\title{
Experimental Study of Packet Loss in a UWB Sensor Network for Aircraft
}

\author{
Daniel Neuhold \\ University of Klagenfurt, Austria
}

\author{
Jorge F. Schmidt \\ University of Klagenfurt, Austria \\ and Lakeside Labs GmbH, Austria
}

\author{
Jirka Klaue \\ Airbus, Hamburg, Germany
}

\author{
Dominic Schupke \\ Airbus, Munich, Germany
}

\begin{abstract}
There is a strong demand in the aviation industry to replace cables in airplanes by wireless connectivity to gain flexibility and reduce weight. Such in-plane wireless communications must be reliable and robust against interference. As part of our activities in this domain, we present a proof-of-concept for an ultra-wideband (UWB) sensor network deployed in a mockup of a small passenger cabin of a commercial aircraft with a few passengers and report experimental results on the packet loss rate with off-the-shelf IEEE 802.15.4-2011 compliant UWB transceivers. It is shown that a combination of spatial and temporal diversity can significantly lower the packet loss rate of different link types without degrading throughput.
\end{abstract}

\section{KEYWORDS}

Ultra-wideband communications, Wireless sensor networks, Aircraft measurements, Reliability, Packet loss, Diversity.

\section{ACM Reference format:}

Daniel Neuhold, Jorge F. Schmidt, Jirka Klaue, Dominic Schupke, and Christian Bettstetter. 2017. Experimental Study of Packet Loss in a UWB Sensor Network for Aircraft. In Proceedings of MSWiM '17, Miami, FL, USA, November 21-25, 2017, 6 pages.

https://doi.org/10.1145/3127540.3127549

\section{INTRODUCTION}

Wireless technologies are rarely used for on-board machine-type communications in today's commercial airplanes. A common fear is that wireless systems cannot compete with traditional wiring in terms of reliability in such critical infrastructure - and indeed, an aircraft cabin is a challenging scenario for wireless communications. To give two examples, the sheer number of people located in a small space results in severe signal shadowing, and the carried wireless devices they carry may cause interference. Despite these concerns and challenges, the industry's need for more cost efficient and greener airplanes is triggering research toward the deployment of in-aircraft wireless networks [9, 11-16]. Use cases

Permission to make digital or hard copies of part or all of this work for personal or classroom use is granted without fee provided that copies are not made or distributed for profit or commercial advantage and that copies bear this notice and the full citation on the first page. Copyrights for third-party components of this work must be honored.

For all other uses, contact the owner/author(s).

MSWiM '17, November 21-25, 2017, Miami, FL, USA

(C) 2017 Copyright held by the owner/author(s).

ACM ISBN 978-1-4503-5162-1/17/11.

https://doi.org/10.1145/3127540.3127549

\author{
Christian Bettstetter \\ University of Klagenfurt, Austria \\ and Lakeside Labs GmbH, Austria
}

include wireless connectivity of hundreds of sensors in passenger cabins and cargo areas.

From an economical perspective, the removal of cables makes planes lighter and has the potential to speed up the production process and lower operating costs $[1,14]$. The deployment flexibility of wireless systems is expected to reduce maintenance costs by avoiding time-consuming repairs in the wiring subject to significant mechanical stress. Beyond this, wireless connections may enable completely new services that are impossible with wires. From the perspective of carbon dioxide emissions, a key factor determining the fuel-saving capabilities of airplanes is their weight. As the fuselage weight is being decreased by the use of lighter hightech materials, the weight of wires in today's airplanes remains fixed, thus becoming a non-negligible fraction $(2-5 \%)$ of the overall weight [1]. To give an example, over $500 \mathrm{~km}$ of wires are deployed in an Airbus A380 [17]. Removing a significant portion of wires is expected to eventually lower emission values.

A key question in this context is: Which technology to use for an in-aircraft wireless sensor network (WSN)? Commonly used WSN solutions (e.g., Zolertia Z1) rely on nodes with low computational power and deliver inadequate data rates. The unlicensed frequency bands used by such technologies are crowded, as most passengers in a typical flight use 802.11 or Bluetooth. Interference in such bands is a major concern in the aviation industry; it may prevent guaranteeing a certain quality of service (QoS).

A promising candidate to tackle these problems is ultra-wideband (UWB) technology. Its high data rates over short distances, highprecision localization, and very low power consumption (a key feature for sustainable operation with harvested energy [6]), together with the fact that current UWB [2] targets frequency bands that do not overlap with mass communication systems, are some features that favor UWB deployment [19]. Moreover, the IEEE 802.15.4-2011 UWB standard contemplates a transmission channel that is likely to comply with in-aircraft wireless regulations. Although the spectrum mask for Wireless Avionics Intra-Communication (WAIC) [1] is not yet defined, a compliance with regard to a characteristic $-5 \mathrm{dBm}$ transmission, with a typical out-of-band attenuation profile, and a bandwidth restriction to $200 \mathrm{MHz}$, seems to be attainable with the IEEE 802.15.4-2011 UWB standard. It must be noted that regulation in some countries (e.g., Australia) forbids UWB operation on board an aircraft, but studies in this domain may contribute to revising these regulations if UWB proves to be a good solution.

We follow this path and deploy an off-the-shelf IEEE 802.15.42011 compliant UWB testbed [5] with up to 17 nodes in a mockup 
of a passenger cabin with a few passengers. Our deployment serves as a proof-of-concept of a WSN in a cabin environment close to reality. It provides first results in network performance accounting for the constraints imposed by commercially available UWB hardware. Different to other experimental contributions in this domain, which mainly focus on channel sounding [13, 15], our objective is to assess the reliability in terms of packet loss achieved by diversity. We first perform a characterization of the propagation effects: we measure the path loss for line-of-sight links (hallway and head panel deployment) and characterize the shadowing for the obstructed links (armrest and floor heights). We also take into account the non-ideal radiation pattern of our antennas. Based on this characterization, we take the perspective of the entire network and analyze simple diversity schemes. We show that the use of code division multiplexing within a time division multiple access (TDMA) scheme, combined with spatial diversity, reduces packet loss without degrading the throughput. The node positioning allows some insight into the system dimensioning.

Our contributions can be summarized as follows: First, we present a proof-of-concept of a UWB-WSN in a passenger cabin mockup of a commercial aircraft. Second, we characterize the path loss and shadowing experienced by commercial UWB nodes in this environment and quantify the loss induced by a single passenger and seat row to be 3 and $6 \mathrm{~dB}$, respectively. Third, we provide packet loss rates for empty and occupied cabins at different flight stages. Fourth, we give insight into the node deployment and system dimensioning.

The paper is organized as follows: Section 2 describes the system, including hardware, network architecture, and multiple access and diversity. Section 3 presents and discusses experimental results on attenuation and packet loss. Section 4 covers related work. Section 5 contains conclusions and outlook to future work. The paper is based on our preliminary results on UWB aircraft sensor networks, presented as extended abstracts and posters $[11,12]$.

\section{SYSTEM}

This study is part of our ongoing efforts to develop a WSN that will replace a number of wired systems in commercial airplanes. We focus on the passenger cabin being the most challenging scenario from the networking perspective. A major challenge is the heterogeneity of system and environment: Applications include seat occupation sensors, security-critical fire and smoke detectors, advanced lighting features, localization of items, and many others. These applications have different priorities and QoS requirements. Some transmissions are periodic, others are event driven. Different parts of the cabin exhibit differences in terms of node density, number of reflexive objects, and degree of mobility.

\subsection{Hardware Platform}

Our network builds on IEEE 802.15.4-2011 UWB compliant transceivers commercialized by DecaWave [5]: the EVK1000 boards (see Fig. 1). The main application and purpose of these boards is indoor localization; they are not calibrated for reliable and efficient data communication. Hence, significant development efforts were needed to adapt these boards to our use case. The newly developed software enables us to meet the aircraft-related QoS requirements and makes off-the-self nodes meet certain system demands. Even

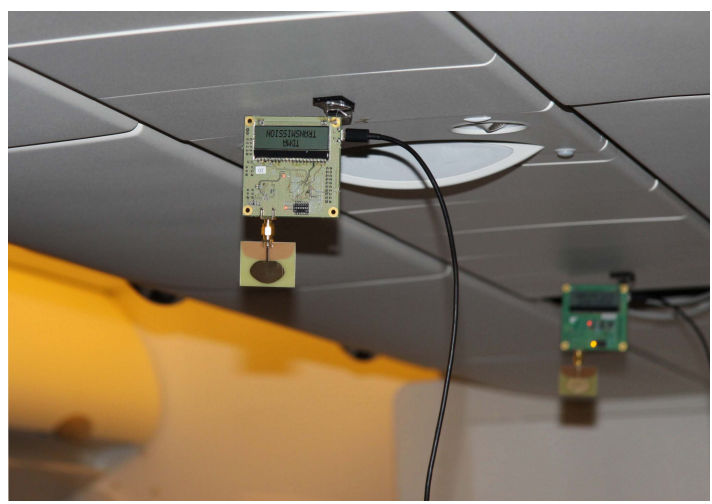

Figure 1: Deployment of DecaWave transceivers in a cabin mockup for test purposes at Airbus in Ottobrunn/Munich.

though different nodes may have different functions, as we employ research kits, all of our nodes consist of the same hardware and differ solely in the software. This enables us to dynamically switch nodes to take over different tasks in different setups.

A characterization of the antennas is needed to ensure that unintended signal attenuation, due to the relative orientation of the antennas, is avoided. Such characterization determines the maximally permitted tilt axes so as not to compromise the quality of the measurements. Our antennas (Fig. 1) exhibit a deep notch on their vertical axis and have maximum gain on their perpendicular direction. Nodes are deployed in a way that the relative antenna orientation is aligned optimally with the main lobe.

\subsection{Network Architecture}

Fig. 2 shows the architecture of the network. An aircraft may have hundreds of sensor nodes, which monitor the environment and report their data to one of several wireless data concentrators (WDCs). The WDCs are connected to the application server, which collects, merges, and ultimately controls the system. There are two types of WDC: access points (APs) and listeners. APs are mounted along the hallway ceiling to establish a cellular network with full coverage of the cabin. They are responsible for controlling, monitoring, and keeping time alignments among nodes and listeners. Listeners are deployed at strategic locations to improve the coverage; they are connected to the APs and provide spatial diversity between nodes and APs. Whenever appropriate, data is sent to the human machine interface for visualization and/or action by the aircraft crew.

\subsection{Multiple Access and Diversity}

2.3.1 Multiple Access. Sensor nodes are expected to rely on energy harvesting to reduce maintenance costs. It is thus of importance to employ protocols that enable duty-cycling and deep-sleep functionality. Furthermore, the aviation industry needs QoS support in terms of reserved channels with requirements on delay and packet loss. Taking this into account, channel resources are arranged into time slots with scheduling performed by the APs. One slot in this time division multiple access (TDMA) scheme is reserved as a common slot to broadcast control information to all nodes. It can be used to notify or reconfigure nodes, e.g., when a 


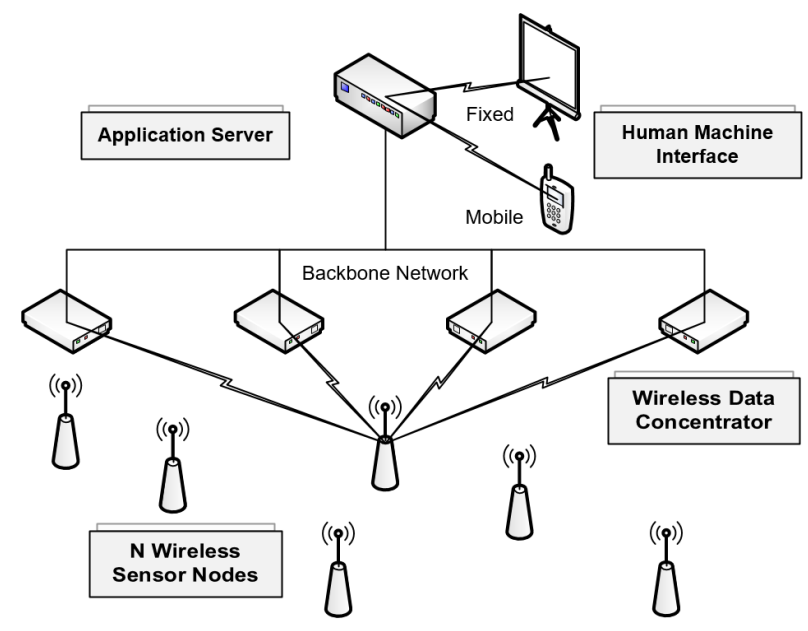

Figure 2: Network architecture.

new node joins the network or when priorities or data rates change. At initialization, each AP executes a node discovery to detect and accommodate nodes in its coverage area. Each node synchronizes its time to its AP. The slot duration is fixed, the TDMA frame scales with the number of nodes, and nodes periodically transmit in their allocated slot. It is assumed that the maximum frame length is short enough to meet the response time targets.

Various factors influence the packet loss, e.g., shadowing and small-scale fading. We investigate as to which types of diversity can be used to efficiently improve the system reliability. We study the benefits of (1) spatial diversity implemented with listeners that overhear transmissions, and (2) temporal diversity in combination with code division multiplexing to enable simultaneous transmissions.

2.3.2 Spatial Diversity. An approach to increase reliability is to add listeners as additional WDCs to provide alternative signal paths. We spatially separate all WDCs as far apart as possible to have independent shadowing conditions. In our experiments, we place one AP in the center of the cabin and four listeners in the corners. We found that this deployment strategy provides both sufficient spatial diversity to overcome fading events and enough alternative receivers to handle the shadowing (see Section 3). With this spatial distribution of receivers, nodes transmit redundant information to all WDCs located at different positions. The cost for this reliability enhancement is an increasing number of WDCs. It is therefore of interest to deploy not more listeners than really needed.

2.3.3 Temporal Diversity. Reliability can be further improvedalternatively, the number of listeners can be reduced - if multiple quasi-orthogonal pseudo noise (PN) codes are used to allow several nodes to transmit in the same slot without message corruption. This is similar to code division multiple access (CDMA), but CDMA cannot be implemented due to limitations of our hardware. Our nodes can be configured to decode a single PN but not all PNs used simultaneously. Because of this limitation, we cannot use the PN to obtain multi-user diversity. Nevertheless, the utilization of different PNs still enables nodes to send redundant messages without reducing the throughput. In our implementation, each listener decodes a different PN, so messages are sent to different WDCs sequentially on each redundant transmission.

As shown in Fig. 3, each node transmits the same message in several consecutive slots altering the PN from slot to slot. This increases the reliability if the channel changes significantly from one slot to the next. Nodes are clustered into groups of size equal to the number $N$ of PNs used. Within groups, nodes alter their PNs in a sequential manner. Redundancy in a factor of $N$ is obtained with each node transmitting periodically in $N$ consecutive slots. In our implementation, we associate each listener to one of these $N=4$ nodes. As nodes change their $\mathrm{PN}$ at each transmission attempt, all nodes transmit their messages to all listeners.

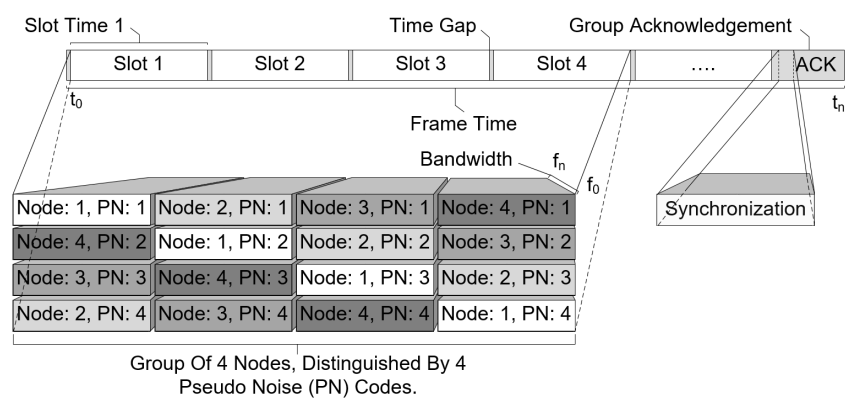

Figure 3: TDMA with code division redundancy for $N=4$.

This scheme can be exploited in fast fading channels. If the channel variation was slow, it is likely that a bad channel condition persists during many slots. We know, however, from our measurements that severe channel fade periods are short compared to the slot duration. The longest fade events are observed during the flight stage when there is very little movement of passengers. In this stage, the combination of spatial and temporal diversity is important.

Note that code division can also be used to increase the data rate instead of reliability. This tradeoff adds flexibility to the design.

\section{EXPERIMENTAL RESULTS}

Two different node deployments in a business class compartment (6.3 m long, $5.1 \mathrm{~m}$ wide) with extended foot room are used to conduct our tests. The first setup is used to characterize the propagation scenario in terms of path loss and shadowing, and gives insight into feasible communication ranges. The second setup is used to evaluate packet loss between nodes and WDCs for realistic deployment positions; it is used to assess the reliability gains of diversity. Small-scale fading is induced by moving passengers.

\subsection{Propagation Features}

We use the testbed setup in Fig. 4 to investigate the attenuation due to path loss and shadowing to estimate the maximum cell size. Shadowing describes the attenuation caused by absorption from objects obstructing the line of sight (LOS) path.

3.1.1 Hallway Test. A fixed AP is deployed in a cabin hallway without passengers (see upper part of Fig. 4) with a transmitting node moving away from the AP as shown. Fig. 5 shows the received power over distance. A path loss exponent of about 2 is found by least square fitting the mean reception power over distance to 


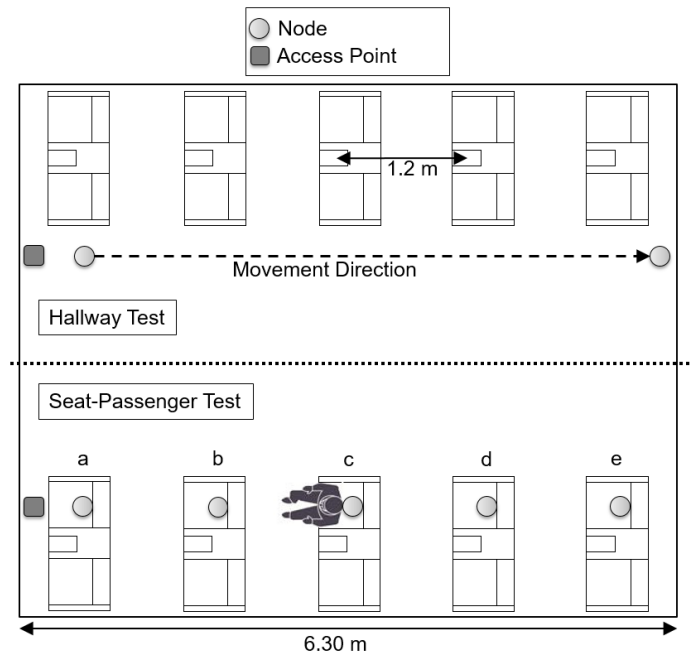

Figure 4: Point-to-point testbed in the hallway and on seats with and without a passenger.

the singular path loss model. There are significant power drops whenever the node passes a seat row in its movement. Since this effect is observed consistently at each seat row, we conjecture that these fluctuations result from the narrow hallway and the small space between the transceiver and the seat when passing by.

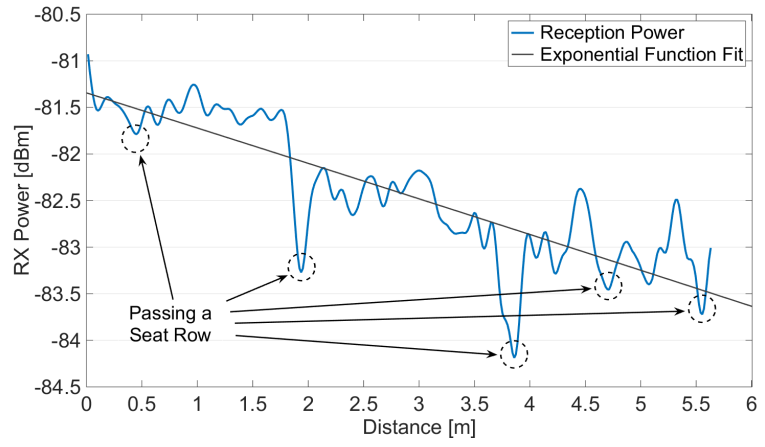

Figure 5: Path loss in a cabin mockup under LOS condition.

3.1.2 Seat-Passenger Test. Let us now quantify the shadowing caused by stationary objects. The majority of objects in a cabin are seats and passengers. The setup is illustrated in the bottom part of Fig. 4. A passenger sits on the right seat in row $c$ for about $220 \mathrm{sec}-$ onds, then stands up and moves out of the cabin. He or she reenters the cabin after 130 additional seconds and repeats the procedure for each other row. Measurements are made on the point-to-point link from the sensor node on the seat to the AP without any other node turned on. Fig. 6 shows that a significant power drop occurs if the signal travels through an occupied seat. When the passenger stands up and leaves the cabin, the signal level increases by $3 \mathrm{~dB}$. This is consistent with the findings in [8] for human body attenuation and complies to our own measurements. The attenuation of a seat

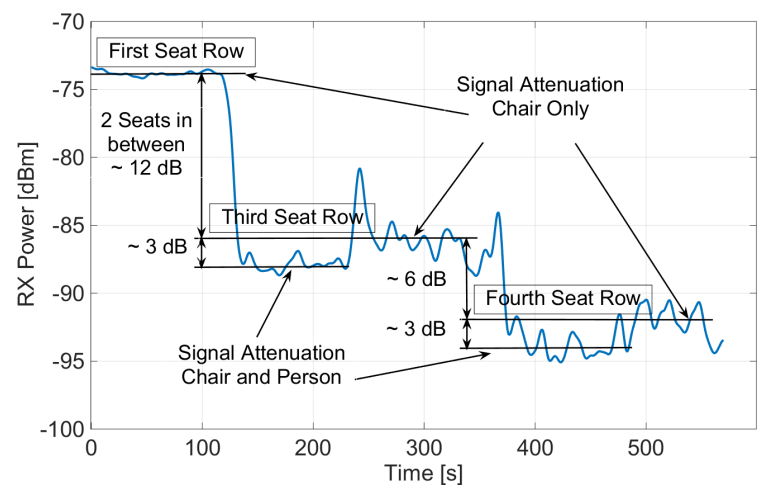

Figure 6: Shadowing in a cabin: Attenuation caused by seats and passengers.

is measured to be $6 \mathrm{~dB}$. As the number of obstructing seat rows increases, the received signal power decreases.

\subsection{Packet Loss}

The packet loss is assessed in a scenario with 12 sensor nodes and 5 WDCs as shown in Fig. 7. There are three nodes on each seat: one at the floor, one at the armrest/seat, and one at the head panel/ceiling. The AP and the four listeners (L1-L4) are deployed at the ceiling. All these positions correspond to practically feasible deployment locations. People in the mockup mimic passengers in different flight phases. A packet loss is accounted for whenever the received signal power falls below the $-105 \mathrm{dBm}$ sensitivity of our nodes.

3.2.1 Impact of Moving Passengers. Passengers obstruct links between senders and receivers, leading to a degraded reception power, which in some cases leads to packet loss. The received power levels are very different to those observed in an empty cabin [12], as shown in Fig. 8 for an armrest node.

3.2.2 Comparison of Deployment Positions. It is of interest for the network design to investigate the impact of the node positioning heights, taking into account that passengers might stand up during boarding, flight, and preparation for de-boarding. Our cabin mockup has almost no packet loss when being empty (see Fig. 9(a)). Packet losses become more frequent if passengers are in the cabin. Fig. 9(b) shows the results in all three flight phases with eight passengers. As passengers take their seats, the packet loss rate increases considerably for nodes at the armrest. This is consistent with our earlier results and is due to the fact that human bodies imply a significant attenuation. As most passengers remain seated during the flight, no major changes are observed for floor and ceiling nodes until the de-boarding phase. Nodes on the floor have a more stable performance. The lowest loss rate occurs for nodes on the ceiling, as most passengers do not obstruct the ceiling links even when walking. The loss rate for the ceiling nodes increases when passengers grab their baggage at the end of the flight. All results are somehow expected in this way; they illustrate the importance of a system that is capable of coping with severe shadowing independently of deployment position or flight stage. 


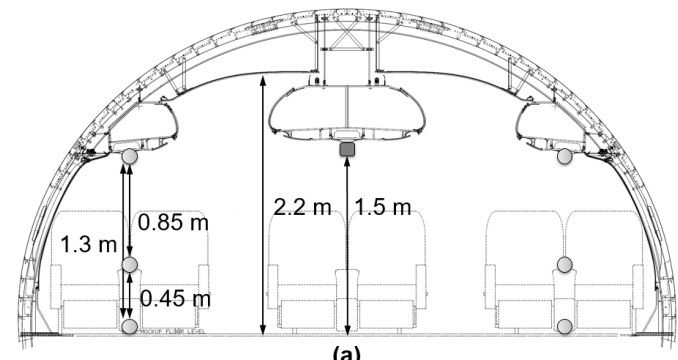

(a)

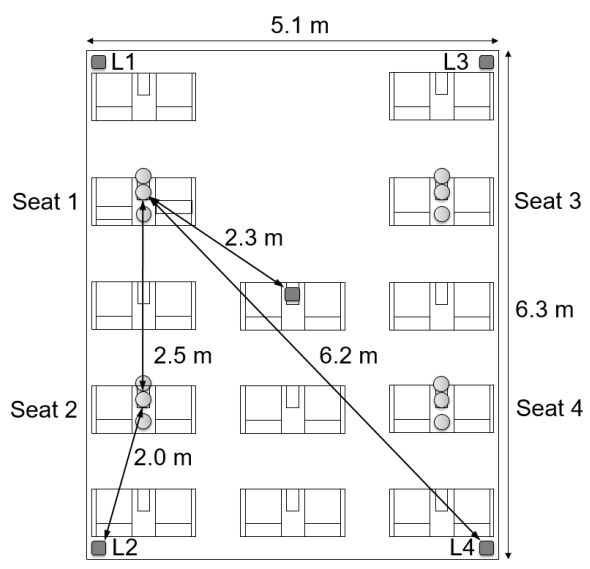

(b)

Figure 7: One-cell testbed with 12 nodes, 1 AP, 4 listeners.

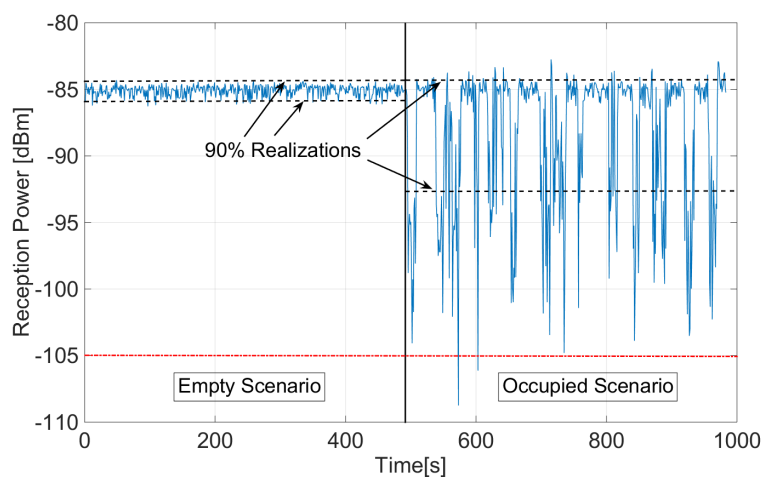

Figure 8: Received signal strength with or without mobility.

3.2.3 Reliability by Diversity. We study as to how spatial and temporal diversity makes the system more reliable. The measurement runs for 30 minutes with eight people moving in the cabin. Their behavior mimics a realistic flight phase and causes link outages. Measurement results are averaged over the deployed nodes independent of their height. Results are shown in Fig. 10. The $x$-axis shows the reception power averaged over all available links.

The top plot shows the packet loss percentage in the TDMA baseline scenario. The loss increases and reaches $100 \%$ well before the average receiver signal strength falls below the receiver sensitivity. In the middle and bottom plots, the stacked bars show the
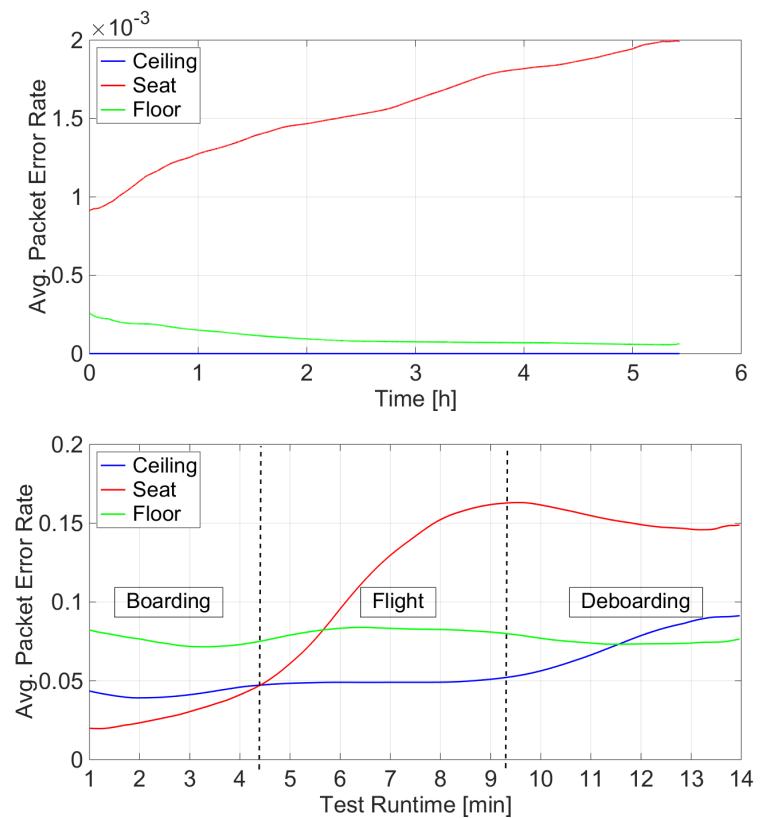

Figure 9: Average packet error rates for different deployment heights in an (a) empty and (b) occupied cabin.

minimum number of listeners needed to successfully recover the lost packets from the top plot. Using spatial diversity (middle plot), the bars show the share of losses that are avoided if using one (dark blue), two (light blue), three (green), or four (yellow) extra listeners, respectively. The same colors are used in the bottom plot, which shows results for the combination of spatial and temporal diversity.

Let us give some examples. Below an average reception power of $-99 \mathrm{dBm}$ almost all packets are lost in the baseline case. Spatial diversity recovers all packet errors up to an average reception power of $-94 \mathrm{dBm}$ by adding one listener. At $-100 \mathrm{dBm}$ almost no packets are recoverable using only one listener, but quite some packets are recoverable with a second listener, and even more with a third one. Finally, as the reception power falls below $-104 \mathrm{dBm}$ (close to sensitivity), packets are only recoverable using four listeners.

Reliability is further improved with the use of temporal diversity implemented as multiple PN codes in addition to spatial diversity (bottom plot). Bars are lower in this case because the use of temporal diversity leads to fewer packet losses to be recovered by adding additional listeners. For example, for an average reception power of $-95 \mathrm{dBm}$ the $40 \%$ packet loss of the baseline scenario is reduced to $20 \%$ due to temporal diversity. This smaller percentage of lost packets determines the need of only one additional listener (bottom) compared to two listeners (middle) to recover all errors at this power level. The movement of passengers is somehow exploited to increase reliability, as an obstructed link is likely to be unobstructed after a short period. Results show that full packet error recovery is reached at minimum reception power of $-86 \mathrm{dBm}$ with no listener (top), $-94 \mathrm{dBm}$ with spatial diversity using only one listener (middle), and $-96 \mathrm{dBm}$ with spatio-temporal diversity and one listener (bottom).

From the design perspective, the limit to the number of redundant codes is given by the cross-correlation properties of the codes 

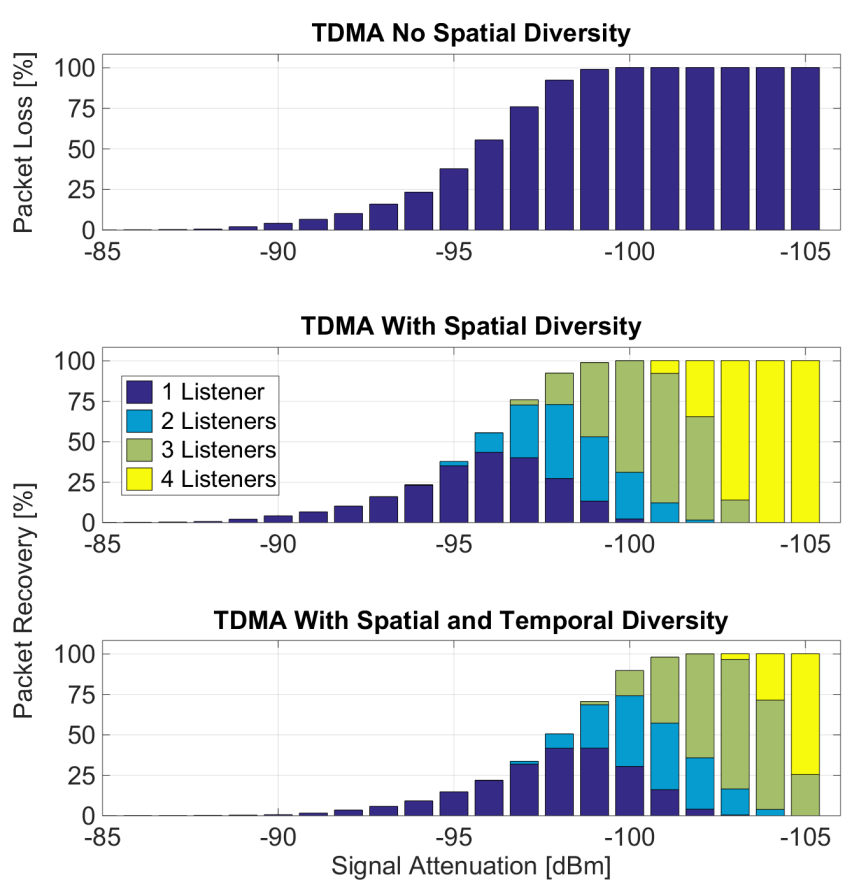

Figure 10: Packet errors for TDMA (top) and improvements by spatial diversity (middle) and spatio-temporal diversity (bottom). An overall number of 600 packets are sent per bar.

and the hardware capabilities. In our case, this yields four different codes. The limit in the degree of spatial diversity is driven by the economical cost of the installation. We can identify that the cell size fixes the average reception power (i.e., the bar to use in Fig. 10). This implies a tradeoff between the number of cells and the number of listeners needed per cell for a given reliability target. The study of this tradeoff is non-trivial and beyond the scope of this paper.

\section{RELATED WORK}

Most related work on UWB focuses on localization [4], tracking [10], and military applications [16]. WSNs for airplanes have been investigated for condition monitoring (e.g., to monitor mechanical parts with special interest on engines $[3,18])$. There are some papers on channel sounding in airplanes. The work in [13] focuses on channel characterizations of UWB in a cabin. These measurements cover a wide range of frequency bands, including the WAIC band. Similar work was done in [15] with a further comparison of cabin and office environments. A main difference to our work is that both mentioned papers are performed with expensive channel sounding equipment, which only allows consecutive measurements to cover different locations in the cabin, and does not account for the effects of practical hardware shortcomings. In this context, the present paper is a continuation of our preliminary work [12] assessing channel features using off-the-shelf transceivers. The influence of passengers was analyzed in [9], where a UWB network was deployed to measure and compare empty and occupied cabins.

\section{CONCLUSIONS AND OUTLOOK}

This paper presented a proof-of-concept for a UWB sensor network in a mockup of an aircraft passenger cabin. Our experimental assessment of reliability demonstrates that spatial and temporal diversity can significantly lower the packet loss rate in this scenario. Full packet error recovery is reached at minimum signal attenuation of $-85 \mathrm{dBm}$ with no listener, $-94 \mathrm{dBm}$ using one listener, and $-100 \mathrm{dBm}$ using three listeners. Temporal diversity reduces the number of required listeners for a given reception power.

Future work will provide results in real and fully-occupied airplanes. The real-time protocol EchoRing [7] is an interesting alternative to our approach and should be investigated in the context of airplanes. From a more general perspective, there is a need for domain-specific interference management and security solutions with low complexity on nodes powered by energy harvesting.

\section{ACKNOWLEDGMENTS}

This work was supported by Airbus, Alpen-Adria-Universität Klagenfurt, and Lakeside Labs $\mathrm{GmbH}$; it was funded by the Carinthian Economic Promotion Fund (KWF) under grant 20214/26481/38805.

\section{REFERENCES}

[1] Technical characteristics and operational objectives for wireless avionics intracommunications (WAIC). Report ITU-R M.2197, Nov. 2010.

[2] Standard for local and metropolitan area networks-Part 15.4: Low-Rate Wireless Personal Area Networks. IEEE Std 802.15.4, Sep. 2011.

[3] H. Bai, M. Atiquzzaman, and D. Lilja. Wireless sensor network for aircraft health monitoring. In Proc. Int. Conf. Broadband Networks, 2004.

[4] K. Balac, M. Akhmedov, M. Prevostini, and M. Malek. Topology optimization of wireless localization networks. In Proc. European Wireless, 2016.

[5] DecaWave UWB-WSN platform. http://www.decawave.com/.

[6] J. M. Dilhac and M. Bafleur. Energy harvesting in aeronautics for battery-free wireless sensor networks. IEEE Aerosp. Electron. Syst. Mag., 29(8):18-22, 2014.

[7] C. Dombrowski and J. Gross. Echoring-meeting hard real-time constraints by decentralized wireless networks. In Proc. NetSys, Cottbus, Germany, Mar. 2015.

[8] I. Dove. Analysis of Radio Propagation Inside the Human Body for in-Body Localization Purposes. Master's thesis, University of Twente, 2014.

[9] M. Jacob, K. L. Chee, I. Schmidt, J. Schuur, W. Fischer, M. Schirrmacher, and T. Kurner. Influence of passengers on the UWB propagation channel within a large wide-bodied aircraft. In Proc. European Conf. on Antennas and Propagation, pages 882-886. IEEE, 2009.

[10] J. Ko et al. Target tracking algorithms for UWB radar network. In Proc. Int. Conf. Radioelektronika, pages 319-324. IEEE, 2016.

[11] D. Neuhold, J. Schmidt, U. Schilcher, G. Brandner, C. Bettstetter, J. Klaue, and D. Schupke. Poster: Towards an ultra-wide band sensor network for aircraft applications. In Prof. ACM Int. Conf. on Embedded Wireless Syst. and Networks (EWSN), pages $217-218,2016$.

[12] D. Neuhold, J. F. Schmidt, C. Bettstetter, J. Klaue, and D. Schupke. Experiments with UWB aircraft sensor networks. In Proc. IEEE INFOCOM Workshops, pages 948-949, April 2016.

[13] H. Saghir, C. Nerguizian, J. Laurin, and F. Moupfouma. In-cabin wideband channel characterization for WAIC systems. IEEE Trans. on Aerospace and Electronic Syst., 50(1):516-529, 2014.

[14] C. Sanchez. Wireless sensor networks on-board aircrafts: Design and implementation of the medium access control protocol. Master's thesis, Universitat Politecnica de Catalunya, 2013.

[15] I. Schmidt, J. Jemai, R. Piesiewicz, R. Geise, M. Schwark, T. Kurner, M. Schirrmacher, and P. Thielker. UWB propagation channels within an aircraft and an office building environment. In Proc. IEEE Int. Symp. Antennas Propagation. IEEE, 2008.

[16] C. Spiliotopoulos and A. Kanatas. Path-loss and time-dispersion parameters of UWB signals in a military airplane. IEEE Antennas Wireless Propag. Lett., 8:790-793, 2009

[17] D. Vujic. Wireless sensor networks applications in aircraft structural health monitoring. Istraz. i Proj. za privredu, 13(2):79-86, 2015.

[18] R. K. Yedavalli and R. K. Belapurkar. Application of wireless sensor networks to aircraft control and health management systems. F. Control Theory Appl., 9(1):28-33, 2011.

[19] J. Zhang, P. Orlik, Z. Sahinoglu, A. Molisch, and P. Kinney. UWB systems for wireless sensor networks. Proceedings of the IEEE, 97(2):313-331, Feb 2009. 